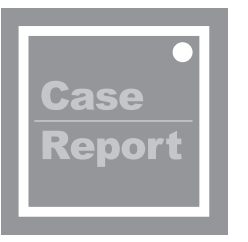

\title{
Endovascular Mechanical Thrombectomy with a Large-Bore Aspiration Catheter and Stent Retriever for Cerebral Venous Sinus Thrombosis in Pregnancy: A Case Report
}

Tsuyoshi Shimizu, ${ }^{1}$ Makoto Sakamoto, ${ }^{1}$ Hidefumi Amisaki, ${ }^{2}$ Yuhei Kuwamoto, ${ }^{1}$ Sadao Nakajima, ${ }^{1}$ Koichi Torihashi, ${ }^{1}$ Tetsuji Uno, ${ }^{1}$ Atsushi Kambe, ${ }^{1}$ and Masamichi Kurosaki ${ }^{1}$

Objective: A case of cerebral venous sinus thrombosis (CVST) during pregnancy effectively recanalized by endovascular mechanical thrombectomy with the combined use of an aspiration catheter and a stent retriever is reported.

Case Presentation: A 27-year-old woman at eight weeks' gestation developed sudden onset of right hemiparalysis and seizures and was referred to our hospital. Her National Institutes of Health Stroke Scale score on admission was 23. On MRI, diffusion-weighted imaging showed a hyperintensity area in the left frontal lobe, and T2* imaging showed hemorrhagic infarction in the same area. MR venography showed obstruction of the anterior two-thirds of the superior sagittal sinus (SSS). Anticoagulant therapy with heparin was started, but since the venous return was expected to be severely impaired, mechanical thrombectomy by endovascular surgery was selected, hoping to resolve symptoms early. Using a large-bore aspiration catheter in combination with a stent retriever, it was possible to safely guide the aspiration catheter into the anterior half of the SSS. The use of a large-bore aspiration catheter enabled retrieval of a large amount of thrombus in a short time, and complete recanalization was achieved. The patient's hemiplegia and aphasia improved significantly within a week after the procedure, and she was discharged without sequelae.

Conclusion: Mechanical endovascular therapy of CVST performed with a combination of a large-bore aspiration catheter and a stent retriever should be considered particularly for patients with severe neurological symptoms or intracranial hemorrhage and for those who do not respond to anticoagulation therapy.

Keywords > cerebral venous sinus thrombosis, endovascular mechanical thrombectomy, endovascular treatment

\section{Introduction}

Cerebral venous sinus thrombosis (CVST) accounts for approximately $0.5 \%-1.0 \%$ of all strokes and is more common in young to middle-aged adults with a fatality rate of $6.0 \%-10 \% .{ }^{1)}$ Conditions associated with CVST can be classified as genetic prothrombotic diseases or acquired factors

${ }^{1}$ Division of Neurosurgery, Department of Brain and Neurosciences, Faculty of Medicine, Tottori University, Yonago, Tottori, Japan ${ }^{2}$ Department of Neurosurgery, Sanin Rosai Hospital, Yonago, Tottori, Japan

Received: September 24, 2021; Accepted: December 4, 2021 Corresponding author: Tsuyoshi Shimizu. Division of Neurosurgery, Department of Brain and Neurosciences, Faculty of Medicine, Tottori University, 36-1, Nishi-cho, Yonago, Tottori 683-8504, Japan Email:msxgsh098@gmail.com

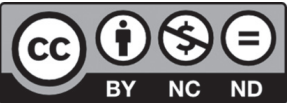

This work is licensed under a Creative Commons Attribution-NonCommercialNoDerivatives International License.

(C)2022 The Japanese Society for Neuroendovascular Therapy such as surgery, trauma, and pregnancy. ${ }^{2)}$ In the acute phase, the primary treatment of CVST is anticoagulant therapy by either unfractionated intravenous heparin or low-molecularweight heparin, but the effectiveness of endovascular treatment (EVT) has been reported in recent years. ${ }^{3}$ A case of CVST occurring in pregnancy that underwent mechanical thrombectomy combined with a large-bore aspiration catheter and stent retriever is presented. The effectiveness of the combined use of a stent and a large-bore aspiration catheter in mechanical thrombectomy is demonstrated, along with a discussion of the previous literature.

\section{Case Presentation}

The patient was a 27-year-old woman (at eight weeks' gestation) who had no medical history and was on no oral medications. She had strong hyperemesis gravidarum (vomiting and anorexia) and could not eat, and she was often asleep in the preceding two weeks. When she was eight weeks pregnant, her family found her lying down in the bathroom and 

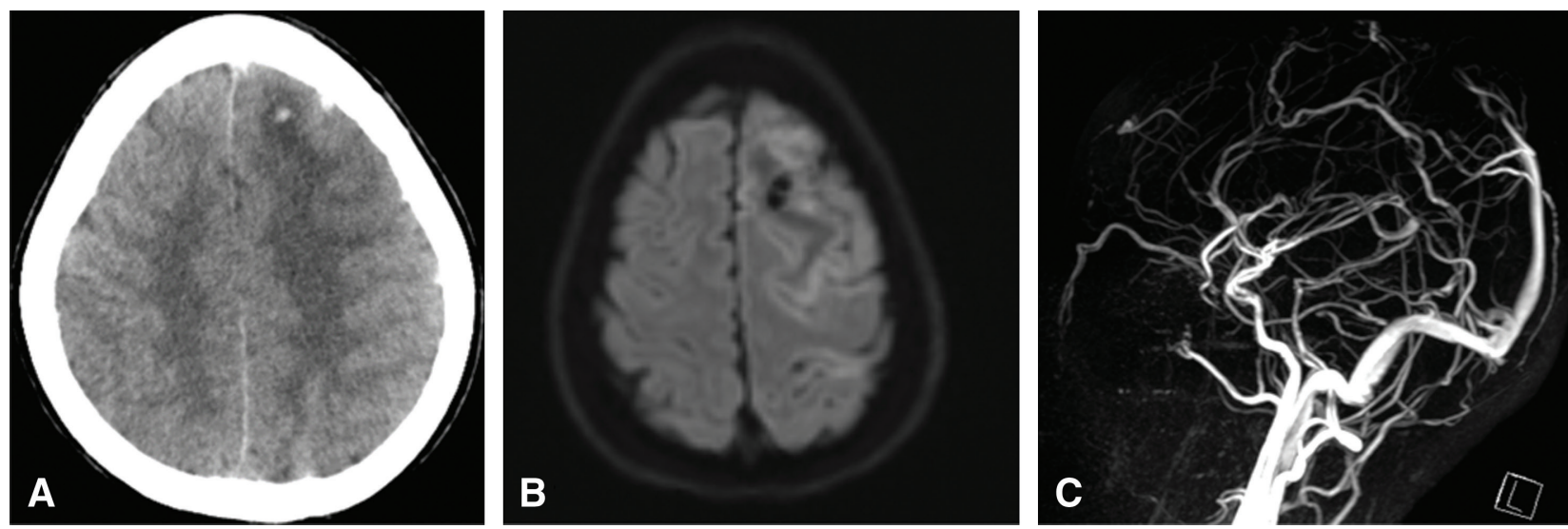

Fig. 1 (A) CT of the brain showing a low-density area in the left frontal lobe with intracerebral hemorrhage. (B) Axial diffusionweighted image of the brain showing high-intensity signals in the left

consulted a local obstetrical hospital. At the local hospital, she developed sudden generalized seizures and was referred to our hospital. On arrival, the Japan Coma Scale was II-10 and the Glasgow Coma Scale was 10 (E4V1M5), and she had right hemiparesis, sensory impairment, motor aphasia, and a National Institutes of Health Stroke Scale score of 23.

On blood testing, the cell counts and general blood chemistry were all in the normal ranges. The coagulation and fibrinolysis system showed a normal prothrombin time (PT; 12.6 seconds), prothrombin time-international normalized ratio (PT-INR; 1.05), and activated partial thromboplastin time (APTT; 28.6 seconds), but D-dimer was slightly increased $(41.3 \mu \mathrm{g} / \mathrm{ml})$. As for the coagulation factors, protein $\mathrm{C}$ was $82 \%$, protein $\mathrm{S}$ was $66 \%$, and antithrombin III was $97 \%$. On immunological testing, antinuclear antibody, myeloperoxidase anti-neutrophil cytoplasmic antibody (MPO-ANCA; $<1.0$ ), proteinase3 anti-neutrophil cytoplasmic antibody (PR3-ANCA; $<1.0$ ), anti-cardiolipin antibody $(<8.0)$, and anti-cardiolipin $\beta 2$ glycoprotein complex antibody $(<1.2)$ were all in the normal ranges.

CT of the head showed a low-density area in the left frontal lobe and small intracranial hemorrhage (Fig. 1A). MRI of the brain showed a hyperintensity area in the left frontal lobe on diffusion-weighted imaging (Fig. 1B), and MR venography showed occlusion of the anterior twothirds of the superior sagittal sinus (SSS) (Fig. 1C).

Since she had generalized seizures and severe neurological symptoms and her symptoms did not improve despite anticoagulation therapy with heparin, it was decided to perform mechanical thrombectomy for early resolution of symptoms.

Mechanical thrombectomy for venous sinus thrombosis was performed under local anesthesia. First, arterial access via the left femoral artery was maintained with a short frontal lobe and a low-intensity area. (C) MR venography showing occlusion of the anterior two-thirds of the SSS. SSS: superior sagittal sinus

sheath (4-French), followed by catheterization of the left internal carotid artery with an angiographic catheter. The venous phase angiogram showed poor visualization of the anterior two-thirds of the SSS (Fig. 2A).

Systemic heparinization was performed by administering 4000 units of heparin. Access to cerebral venous vasculature was obtained with a shuttle long sheath (7-French, length $80 \mathrm{~cm}$ ) via the right femoral vein, and the tip of the sheath was advanced into the left internal jugular vein in combination with a Countdown 6 catheter (Medikit, Tokyo, Japan) and Fukuda Guide wire 0.035-150 cm (Fukuda Denshi, Tokyo, Japan).

First, the Catalyst 6 aspiration catheter (inner diameter of 0.060 inch; Stryker, Kalamazoo, MI, USA) was navigated into the proximal one-third of the SSS with a Phenom 27 microcatheter (Medtronic, Minneapolis, MN, USA) and CHIKAI-14 (ASAHI INTECC, Aichi, Japan), and then the Catalyst 6 was gently advanced to near the occluded segment.

After removing the Phenom 27 and CHIKAI-14, manual aspiration from the Catalyst 6 could be done, but only small amounts of thrombus were removed. Although manual aspiration was performed three times, the thrombus could be removed only in small amounts, so it was decided to use a larger bore aspiration catheter. A React 71 aspiration catheter (inner diameter of $0.071 \mathrm{inch}$; Medtronic) was guided to near the thrombus in the same manner and connected to the aspiration pump, and then a large amount of thrombus could be removed continuously. The React 71 was advanced while aspirating the clot bit by bit, but when it reached the anterior one-third of the SSS, the ledge of the aspiration catheter was caught in the outer wall of the venous sinus, making it difficult to advance. Therefore, it was decided to deploy the stent in the thrombus-occluded SSS first and 

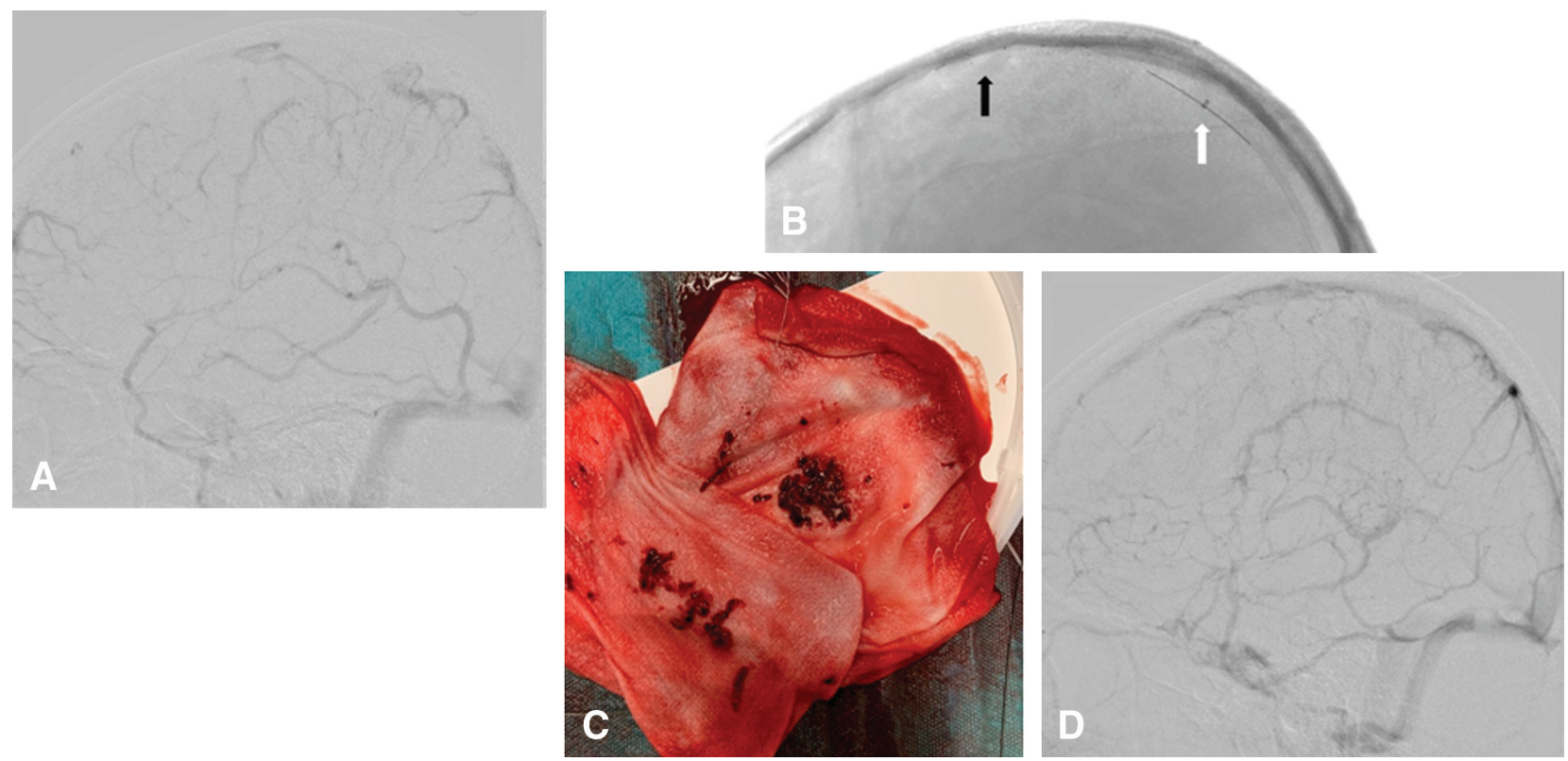

Fig. 2 (A) Lateral view of the preoperative left carotid angiography showing occlusion of the anterior two-thirds of the SSS. (B) A stent retriever (Solitaire $6 \times 40 \mathrm{~mm}$ ) is deployed (black arrow), and the aspiration catheter (React 71) is advanced along with the stent retriever (white arrow). (C) Mechanical thrombectomy is performed

then advance the aspiration catheter along it to reduce the trapping of the aspiration catheter by the ledge.

A Solitaire stent retriever $6 \times 40 \mathrm{~mm}$ (Medtronic) was deployed across the occluded sinus, and after removing the Phenom 27, the React 71 was aligned with the previously deployed Solitaire axis and advanced along with it, applying suction (Fig. 2B).

By retracting the stent with the thrombus into an aspiration catheter while aspirating from a large-bore aspiration catheter, a large amount of thrombus in the SSS could be removed (Fig. 2C). The venous phase of the final cerebral angiogram showed that the SSS was completely reopened, and cerebral circulation was markedly improved (Fig. 2D).

The total radiation absorbed dose was $432 \mathrm{mGy}$, and the total use of iodinated contrast agents was $50 \mathrm{ml}$ of iopamidol.

Systemic heparinization was maintained by continuous heparin administration of 10000 to 15000 units per day. After through discussions with her and her family about the continuation of pregnancy, she decided to have an abortion. In collaboration with obstetricians and gynecologists, an abortion was performed 20 days after the procedure.

Then, 21 days after the procedure, heparin anticoagulation was switched to oral warfarin therapy. MRI 22 days after the procedure showed no new cerebral infarction or bleeding, and MR venography showed complete recanalization of the SSS.

The patients' symptoms were rapidly improved by rehabilitation, and five weeks later, she had no neurologic using a stent retriever combined with an aspiration catheter, and a large amount of crushed red and black clots are removed from the stent retriever and the aspiration catheter. (D) Lateral view of the postoperative angiography showing recanalization of the SSS. SSS: superior sagittal sinus

symptoms and was discharged home with a modified Rankin Scale (mRS) score of 0.

\section{Discussion}

CVST is a relatively rare disease, accounting for $0.5 \%$ $1.0 \%$ of all strokes, and it occurs commonly in young to middle-aged people. In many cases of CVST, there are various clinical symptoms, such as intracranial hypertension (vomiting, nausea, headache, etc.), seizures, and focal neurological deficits. According to some reports, the prognosis of CVST is usually good, and mortality from CVST is $3 \%-15 \%$, with a peak within the first 30 days.

Systemic anticoagulation has been regarded as the first-line treatment, while EVT, such as mechanical thrombectomy or direct thrombolysis, should be considered in patients with clinical deterioration or progression of intracerebral hemorrhage or venous infarction despite anticoagulation therapy. ${ }^{4}$

EVT is also recommended in the current guide for CVST in patients with a high risk of poor outcome (prognostic score for CVST [malignancy, coma, deep venous thrombosis, mental status disturbances, male sex, intracranial hemorrhage] 23). ${ }^{5)}$ In the present case, EVT was performed because the patient was classified to be of high risk with score 4 .

In recent years, there have been some reports that EVT for patients with CVST has provided good clinical outcomes and high successful recanalization rates. . $^{1,3,67)}$ Currently 
available EVTs include catheter thrombolytic therapy using a thrombolytic agent, balloon-assisted angioplasty, rheolytic catheter thrombectomy using AngioJet (Possis Medical, Minneapolis, MN, USA), aspiration thrombectomy, and stent retriever thrombectomy. In recent years, with the rapid evolution of stents and aspiration catheters used in mechanical thrombectomy for acute ischemic stroke, there has been an increasing number of reports of thrombus retrieval for venous sinus thrombosis using these devices. The use of mechanical thrombectomy offers several theoretical advantages over medical therapy and local endovascular thrombolysis because it can achieve direct and rapid recanalization without the use of fibrinolytic agents in cases with intracerebral hemorrhage.

In addition, the procedure can be performed in the same way as for an acute ischemic stroke, and devices are always available in the hospital, so that it can be performed immediately.

In a systematic review of 213 patients with CVST who underwent EVT, Ilyas et al. reported complete recanalization in $69 \%$, an mRS score of $0-2$ in $76 \%$, and that a good clinical outcome was significantly associated with early treatment $^{3}$ ) (odds ratio [OR] 0.12, 95\% confidence interval [CI] $0.02-0.86, p=0.13]$. They also reported that worsening or new intracranial hemorrhage occurred in $8.7 \%$ of cases, which was not significantly associated with EVT (OR 1.15, 95\% CI 0.12-10.80, $\mathrm{p}=0.90$ ). They reported that local thrombolytic therapy was used in more than $30 \%$ of cases, and thrombus retrieval using stents or aspiration catheters was used in only about $20 \%$ of cases, suggesting that the use of these devices may improve treatment outcomes.

There are some reports that showed the effectiveness of EVT for CVST with intracranial hemorrhage. ${ }^{1,8)}$ Guo et al. ${ }^{8)}$ performed EVT (41 cases of local thrombolysis and 15 cases of mechanical thrombectomy) for 56 CVST patients with intracranial hemorrhage and found that $87.5 \%$ had good neurological recovery (mRS score 0-2) at discharge. Of the 51 cases that were followed up, $96 \%$ of patients (49) had an mRS score of $0-2$ at 6 months.

In a series by Styczen et al.," ${ }^{7)}$ EVT was performed for 13 patients with CVST who did not respond to anticoagulant therapy; $86 \%$ of patients had successful recanalization, and $92 \%$ of patients had an mRS score of $0-2$ at the last follow-up. Possible indications for mechanical thrombectomy included failure of systemic anticoagulation, extensive thrombus burden, cerebral edema, increased intracranial pressure, and progressive worsening of neurologic symptoms.

The Thrombolysis or Anticoagulation for Cerebral Venous Thrombosis trial was recently published, ${ }^{9}$ and the patients were randomized to receive either EVT with standard medical care (EVT group $[\mathrm{n}=33]$ ) or standard medical care only (control group $[\mathrm{n}=34]$ ).

At the 12-month follow-up, mRS score of 0-2 (85\% vs $82 \%$, relative risk ratio $1.03,95 \%$ CI $0.83-1.27)$, mortality rate $(12 \%[n=4]$ vs $3 \%[n=1], p=0.2)$, and the frequency of systemic intracranial hemorrhage $(3 \%[\mathrm{n}=1]$ vs $9 \%$ $[\mathrm{n}=3], \mathrm{p}=0.61$ ) were not significantly different between the two groups. However, this trial had several limitations. First, this trial included only 67 patients and was therefore underpowered to detect a small difference between the two groups. Second, the mainstay of treatment was AngioJet $(\mathrm{n}=14,42 \%)$, followed by chemical thrombolysis $(\mathrm{n}=17$, $52 \%)$, with a stent retriever used in only 5 cases $(15 \%)$.

In the present case, thrombus was relatively widespread in the anterior two-thirds of the SSS, so direct aspiration was performed several times using a Catalyst 6 aspiration catheter first. However, the Catalyst 6 quickly became obstructed by a large amount of clot, and it was necessary to remove the catheter from the patient, and then reopen it and reinsert it, which required time to perform aspiration again. Therefore, a combined technique with a Solitaire $6 \times 40 \mathrm{~mm}$ stent and a React 71, which is the largest bore catheter currently available in Japan, was performed, and complete recanalization was achieved.

A combined technique with a stent retriever and an aspiration catheter (stent anchor with mobile aspiration technique) for CVST was first reported by Raychev et al. in 2014. ${ }^{10}$

There have been some reports treated with the combined technique captured thrombus by both the stent retriever and the aspiration catheter ${ }^{7,11)}$ as in the present case, and it is considered an effective treatment method. In addition, there have been some reports of the combined use of aspiration thrombectomy and intrasinus thrombolysis using a penumbra aspiration catheter. ${ }^{12,13)}$

Aspiration thrombectomy with a large-bore aspiration catheter using REACT 71 was reported by Sundar et al., ${ }^{14)}$ and may be a safe and effective treatment method for CVST.

If the risk of hemorrhagic complication is higher, mechanical thrombectomy with a stent retriever, a largebore aspiration catheter, or a combination of them may be recommended without the use of thrombolytic agents.

In the present case of CVST developed during pregnancy, endovascular mechanical thrombectomy with systemic anticoagulant therapy was performed, which provided a good clinical outcome. There was no thrombophilia on blood tests, and she had strong hyperemesis gravidarum, suggesting that the cause of the CVST was pregnancy. Although 
there is no consensus on the treatment of CVST during pregnancy, Kashkoush et al. ${ }^{15)}$ reported that a combination of anticoagulant therapy with endovascular mechanical thrombectomy $(n=5)$ or intra-arterial thrombolysis $(n=17)$ for patients in pregnancy and the puerperium resulted in a good clinical outcome ( $\mathrm{mRS}$ score $0-2)$ in $82 \%(\mathrm{n}=18)$.

According to the International Commission on Radiological Protection, termination of pregnancy at fetal doses of less than $100 \mathrm{mGy}$ is not justified based upon radiation risk. ${ }^{16)}$ Tanaka et al. ${ }^{17)}$ reported that the estimated fetal mean radiation dose is approximately $0.05 \mathrm{mGy}$, when the maternal head maximum exposure is approximately $800 \mathrm{mGy}$. Her total radiation absorbed dose was $432 \mathrm{mGy}$, so fetal exposure dose was expected to be lower than this dose.

Finally, the treatment of patients with CVST in the chronic phase is described. Warfarin is often the treatment of choice in the chronic phase, but whether direct oral anticoagulant (DOAC) therapy is an acceptable treatment choice of treatment for these patients is not clear.

The result of the recent RE-SPECT CVT trial (a clinical trial comparing the efficacy and safety of dabigatran etexilate with warfarin in patients with CVST and dural sinus thrombosis), which compared dabigatran $150 \mathrm{mg}$ bid versus doseadjusted warfarin for systemic CVST, ${ }^{18)}$ showed no difference in the recurrence of venous thrombosis between the two groups, and major bleeding was less in the dabigatran group $(1.7 \%$ vs $3.3 \%)$. Although there are few reports of DOAC use for patients with CVST, and the clinical outcome is excellent, ${ }^{19)}$ it is possible that DOACs are effective from the results of the RE-SPECT CVT trial. It is a treatment method that may be considered for young women, as in the present case.

\section{Conclusion}

Endovascular mechanical thrombectomy for CVST is a safe and effective treatment, which can be one of the good treatment options, especially in patients with a severe neurological deficit or with an intracranial hemorrhage, and for those who do not respond to systemic anticoagulation therapy.

A combined technique with a large-bore aspiration catheter and a stent retriever was effective, good recanalization was achieved, and the prognosis was good. Accumulation of further cases is an issue for the future.

\section{Disclosure Statement}

The authors declare that they have no conflicts of interest associated with this manuscript.

\section{References}

1) Zhang S, Hu Y, Li Z, et al. Endovascular treatment for hemorrhagic cerebral venous sinus thrombosis: experience with 9 cases for 3 years. Am J Transl Res 2018; 10: 1611-1619.

2) Shindo A, Wada H, Tomimoto H. Cerebral venous thrombosis. J Thromb Haemost 2014; 25: 399-403. (in Japanese)

3) Ilyas A, Chen CJ, Raper DM, et al. Endovascular mechanical thrombectomy for cerebral venous sinus thrombosis: a systematic review. J Neurointerv Surg 2017; 9: 1086-1092.

4) Lee SK, Mokin M, Hetts SW, et al. Current endovascular strategies for cerebral venous thrombosis: report of the SNIS Standards and Guidelines Committee. J Neurointerv Surg 2018; 10: 803-810.

5) Ulivi L, Squitieri M, Cohen H, et al. Cerebral venous thrombosis: a practical guide. Pract Neurol 2020; 20: 356-367.

6) Goktay AY, Senturk C: Endovascular treatment of thrombosis and embolism. Adv Exp Med Biol 2017; 906: 195-213.

7) Styczen H, Tsogkas I, Liman J, et al. Endovascular mechanical thrombectomy for cerebral venous sinus thrombosis: a single-center experience. World Neurosurg 2019; 127: e1097-e1103.

8) Guo XB, Liu S, Guan S. The clinical analysis and treatment strategy of endovascular treatment for cerebral venous sinus thrombosis combined with intracerebral hemorrhage. Sci Rep 2020; 10: 22300. https://doi.org/10.1038/s41598-020-78570-1

9) Coutinho JM, Zuurbier SM, Bousser MG, et al. Effect of endovascular treatment with medical management vs standard care on severe cerebral venous thrombosis: the TO-ACT randomized clinical trial. JAMA Neurol 2020; 77: 966-973.

10) Raychev R, Tateshima $S$, Rastogi $S$, et al. Successful treatment of extensive cerebral venous sinus thrombosis using a combined approach with Penumbra aspiration system and Solitaire FR retrieval device. J Neurointerv Surg 2014; 6: e32.

11) Taniguchi S, Harada K, Kajihara M, et al. Combined use of stent-retriever and aspiration thrombectomy for cerebral venous sinus thrombosis involving the straight sinus: a case report. Interv Neuroradiol 2017; 23: 605-608.

12) Tsang ACO, Hwang AC, Chiu RHY, et al. Combined aspiration thrombectomy and continuous intrasinus thrombolysis for cerebral venous sinus thrombosis: technical note and case series. Neuroradiology 2018; 60: 1093-1096.

13) Medhi G, Parida S, Nicholson P, et al. Mechanical thrombectomy for cerebral venous sinus thrombosis: a case series and technical note. World Neurosurg 2020; 140: 148-161.

14) Sundar K, Paulraj S, Choudhury SR, et al. Successful endovascular treatment of cerebral venous thrombosis with a novel, larger aspiration catheter (REACT): a case report. Neurointervention 2021; 16: 83-87.

15) Kashkoush AI, Ma H, Agarwal N, et al. Cerebral venous sinus thrombosis in pregnancy and puerperium: a pooled, systematic review. J Clin Neurosci 2017; 39: 9-15. 
Shimizu T, et al.

16) International Commission on Radiological Protection. The 2007 Recommendations of International Commission on Radiological Protection. ICRP Publication 103. UK: Pergamon Press, Oxford, 2007.

17) Tanaka T, Sadatoh A, Hayakawa M, et al. Endovascular treatment of stroke during pregnancy: measuring the radiation exposure dose of lower abdomen using human body phantom. JNET J Neuroendovasc Ther 2013; 7: 243-251. (in Japanese)
18) Ferro JM, Coutinho JM, Dentali F, et al. Safety and efficacy of dabigatran etexilate vs dose-adjusted warfarin in patients with cerebral venous thrombosis: a randomized clinical trial. JAMA Neurol 2019; 76: 1457-1465.

19) Geisbüsch $C$, Richter $D$, Herweh $C$, et al. Novel factor xa inhibitor for the treatment of cerebral venous and sinus thrombosis: first experience in 7 patients. Stroke 2014; 45: 2469-2471. 